\title{
The Value of Coronary Artery Calcium Scoring in Early Diagnosis of Radiation-Induced Coronary Artery Disease in Breast Cancer Patients Following Radiation
}

\author{
Abbas Arjmand Shabestari, ${ }^{1}$ Robab Anbiaee, Taraneh Faghihi Langroudi, ${ }^{1}$ Hooman Bakhshandeh, ${ }^{1}$ \\ and Maryam Heydari-Sooreshjaany ${ }^{1}$ \\ ${ }^{1}$ Shahid BeheshtiUniversity of Medical Sciences,Tehran, Iran \\ Received 2016 December 21; Accepted 2017 February 08.
}

\begin{abstract}
Background: Breast cancer $(\mathrm{BC})$ mortality rate has significantly decreased during the past decades by introducing adjuvant chemotherapy and/or radiotherapy (RT) in addition to surgery. However, as an emerging phenomenon, the corresponding therapeutic side effects caused non-malignant morbidity and mortality. Coronary artery disease (CAD) is the leading non-malignant cause of death in BC patients. It is established that chest RT has a role in atherosclerotic plaque formation in the coronary arteries. Newer techniques of RT like three-dimensional conformal radiotherapy (3D-CRT) use technical methods in which less radiation doses are given to the non-cancerous tissues. Non-invasive technique of coronary artery calcium score (CACS) can detect atherosclerotic plaques long before the plaques become symptomatic, helping to treat them in early stages, so improve BC patients' outcome. The aim of this study is to assess and compare CACS in BC patients with a history of 3D-RT and non-BC women in the same age range with no history of RT.

Methods: CACS of fifty BC patients (age range: 34 - 70 years) with different time intervals from RT (range: 3-9 years) and CACS of fifty age-matched control group of non-BC women with no history of chest RT, using 64-slice ECG-gated CT scan were calculated and presented as the quantitative value of Agatston score (AS). The risk factors contributing to CAD (hypertension, hyperlipidemia, diabetes mellitus, smoking), and the Framingham's 10-year risk score, as well as the age-matched CACS percentiles, were evaluated. Results: No correlation between AS and history of RT, RT-to-follow-up time interval, laterality of BC, Framingham's 10-year risk score or traditional CAD risk factors were detected. Increase in CACS related to the senile atherosclerotic process was shown $(\mathrm{P}<0.001)$. Conclusions: No difference between CACS was noted between BC patients with a history of 3D-CRT and the non-BC women without a history of RT. Although cardiac radiation dose reduction in 3D-CRT technique can justify the findings, as multiple studies have shown increase of CAD in BC patients with history of old RT techniques, but some studies show no difference in CACS between new and old RT techniques, other reason would be non-calcified atherosclerotic plaques after RT or myocardial microvascular disease due to reduced cardiac doses after 3D-CRT rather than calcified atherosclerotic plaques seen naturally by aging.
\end{abstract}

Keywords: Breast Cancer, Three-Dimensional Conformal Radiotherapy, Calcium Score, Coronary Artery Disease

This is an abstract presented in the 33rd Iranian congress of radiology (ICR) and the 15th congress of Iranian radiographic science association (IRSA) 